\title{
Detection of Enterovirus RNA in Peripheral Blood Mononuclear Cells of Type 1 Diabetic Patients Beyond the Stage of Acute Infection
}

\author{
Barbara M. Schulte,, Judith Bakkers, ${ }^{1}$ Kjerstin H.W. Lanke, Willem J.G. Melchers, \\ Ciska Westerlaken, ${ }^{2}$ Wil Allebes, ${ }^{3}$ Henk-Jan Aanstoot, ${ }^{4}$ G. Jan Bruining, ${ }^{5}$ \\ Gosse J. Adema, ${ }^{6}$ Frank J.M. Van Kuppeveld, and Jochem M.D. Galama ${ }^{1}$
}

\begin{abstract}
Previous studies have shown that enteroviral RNA can be detected in blood at the onset of type 1 diabetes (T1D). The infection may play a role in triggering T1D and genetic host factors may contribute to this process. We investigated (1) whether enterovirus is present at the onset of T1D in peripheral blood mononuclear cells (PBMC), plasma, throat, or stool, and (2) whether enteroviral presence is linked with HLA-DR type and/or polymorphisms in melanoma differentiation-associated gene 5 (MDA5) and 2'-5' oligoadenylate synthetase 1 (OAS1), factors of antiviral immunity. To this end, PBMC, plasma, throat, and stool samples from 10 T1D patients and 20 unrelated controls were tested for the presence of enteroviruses (RT-PCR), for HLA-DR type, and polymorphisms in MDA5 and OAS1. Enterovirus RNA was detected in PBMC of 4/10 T1D patients, but none of 20 controls. Plasma was positive in 2/10 T1D patients and none of 20 controls, suggesting that enteroviruses found at the onset of T1D are mainly present in PBMC. All throat samples from positive T1D patients were virusnegative and only 1 fecal sample was positive. The negative results for all throat and most stool samples argues against acute infection. Enterovirus presence was linked with HLA-DR4, but not with polymorphisms in MDA5 or OAS1.
\end{abstract}

\section{Introduction}

$\mathrm{C}$ OXSACKIE B viruses AND ECHOviruses, both belonging to the human enterovirus B group, have for decades been implicated as environmental factors in type 1 diabetes (T1D), which is generally regarded as an autoimmune disease (reviewed in 13 and 24). Support for their role in T1D is deduced from: (1) prospective cohort studies that showed that enterovirus infections coincide with onset of autoimmunity and with clinical onset of T1D (13); (2) detection of enterovirus RNA in blood of T1D patients (see below); and (3) detection of enterovirus in islets of individuals with established T1D $(10,23,33)$. Thus enteroviruses may be involved in T1D via various routes: indirectly, through triggering of autoimmunity, or more directly, through infection of islets and de- struction of $\beta$ cells. It should be noted, however, that these two processes are not necessarily mutually exclusive; they may even be interrelated.

Enterovirus RNA has been found in blood by RT-PCR at onset of disease in $20-75 \%$ of T1D patients $(1,3,5,6,8,17$, 18,32). Enterovirus has furthermore been found in autoantibody-positive (pre-diabetic) persons (15-17), and in patients with established T1D $(1,3)$, but not, or with a much lower frequency, in matched healthy controls without predisposition for T1D or in T2D patients (1). So far, none of the studies that detected viral RNA in the blood of T1D patients investigated the stage of infection, or whether a positive RT-PCR result was due to early infection with virus excreted in the throat, or due to convalescence with virus detectable in stool only, which can last for up to 3 mo (4). Another possibility

\footnotetext{
${ }^{1}$ Department of Medical Microbiology, and ${ }^{6}$ Department of Tumor Immunology, Radboud University Nijmegen Medical Centre and Nijmegen Centre for Molecular Life Sciences, Nijmegen, The Netherlands.

${ }^{2}$ Canisius Wilhelmina Hospital, Nijmegen, The Netherlands.

${ }^{3}$ Department of Bloodtransfusion and Transplantation Immunology, Radboud University Nijmegen Medical Centre, Nijmegen, The Netherlands.

${ }^{4}$ Diabeter, Center for Pediatric and Adolescent Diabetes Care and Research, Rotterdam, The Netherlands.

${ }^{5}$ Department of Pediatrics, Erasmus University Medical Centre, Rotterdam, The Netherlands.
} 
is that virus remains detectable beyond convalescence (stoolnegative). Taken together, this difference from unrelated healthy people suggests that T1D patients and those at risk for the disease are more susceptible to enteroviruses, or are unable to clear the infection, both conditions that may be involved in T1D.

Besides environmental factors, a strong genetic predisposition is involved. HLA-DR3 and DR4 are major risk factors, whereas DR2 is negatively associated with the disease $(2,7$, $20,22,29)$. Other genetic factors have also been associated with T1D, and new associations are still being found, including two recently identified single nucleotide polymorphisms (SNPs) that are involved in antiviral immunity $(11,26,28,30)$. These factors, melanoma differentiation-associated gene 5 (MDA5) and $2^{\prime}-5^{\prime}$ oligoadenylate synthetase 1 (OAS1), play an important role in the innate defense against RNA viruses by sensing or degrading enteroviral RNA, respectively. These SNPs may thus provide a link between enteroviruses and T1D.

In this study we investigated (1) whether peripheral blood mononuclear cells (PBMC) contain enteroviral RNA, (2) what is the stage of infection, and (3) whether infection is linked to particular risk alleles for HLA-DR and/or with SNPs in MDA5 and OAS1.

\section{Patients and Methods}

\section{Patients and control group}

Patients and controls were from the Paediatric Clinics of the Erasmus University Medical Centre, Rotterdam and the Canisius-Wilhelmina Hospital, Nijmegen. Ten T1D patients (5 males and 5 females) were included and samples were collected within a month of diagnosis. Mean age of the patient group was 9.7 y (range 5-14 y). For controls, a group of 20 hospitalized children ( 9 males and 10 females, 1 of whom for which the gender was unknown) of the same age range with non-endocrine disorders was included. Their mean age was 12.5 y (range 6-17 y). Samples from the T1D patients were obtained between November 2003 and November 2004, and from healthy controls in June 2004. Informed consent was given by the parents, based on a study protocol that was approved by the local ethical committees of the respective hospitals.

\section{Laboratory samples}

The following samples were collected: (1) $5 \mathrm{~mL}$ of citrate blood for isolation of plasma and PBMC, (2) $5 \mathrm{~mL}$ of clotted blood for serum, (3) a throat swab in virus transport medium, and (4) a fecal sample. PBMC were isolated as instructed by the manufacturer (Becton and Dickinson, Erembodegem, Belgium). After processing, all samples were flash frozen and stored at $-80^{\circ} \mathrm{C}$ until use.

\section{Virus detection}

RNA was isolated from $200 \mu \mathrm{L}$ of plasma, the equivalent of $2 \times 10^{5}$ PBMCs (isolated PBMCs dissolved in PBS), and stool sample homogenates. For the in-house nested RT-PCR, RNA extraction was done using the MagNaPure LC Isolation station (Roche Applied Science, Mannheim, Germany). Amplification was performed in a nested PCR reaction as previously described (9), as was real-time PCR (19). Real-time NASBA (NucliSens EasyQ) was performed according to the manu- facturer's instructions (BioMérieux, Boxtel, The Netherlands). The amplification techniques were regularly tested against Quality Control for Molecular Diagnostics (QCMD) standards. An enterovirus-specific internal homologous control RNA was included in each sample during the extraction to monitor the NucliSens EasyQ and PCR procedures at the individual sample level. This excludes false-negatives due to inhibitory factors in the input samples, which may be present, particularly in stool samples. The sensitivity of the methods is at least $0.002-0.01 \mathrm{TCID}_{50}$ for in-house nested RT-PCR and real-time PCR, depending on the enterovirus strain tested. NASBA is similarly or somewhat less sensitive than the PCR, depending on the enterovirus strain.

\section{Interferon- $\alpha$ detection}

The amount of interferon- $\alpha$ (IFN- $\alpha)$ in the plasma samples was determined using a commercial ELISA kit, product number RDI-PB41110, from Research Diagnostics Inc. (Fitzgerald Industries International, North Acton, USA). The test was performed according to the manufacturer's instructions.

\section{HLA typing}

Total nucleic acids (NA) was isolated using MagNaPure as described above. HLA typing was performed on RNasetreated DNA using the lifecodes HLA-SSO typing kit (Tepnel, Manchester, UK) according to manufacturer's instructions and EFI/ASHI guidelines for histocompatibility testing.

\section{MDA5 and OAS1 single nucleotide polymorphism typing}

The MDA5 SNP tested was the A946T polymorphism (rs1990760) described by Smyth et al. (26). The OAS1 SNP tested was rs10774671, which is an A/G splice-site SNP $(11,27,28)$. SNPs in MDA5 and OAS1 were assessed by amplification of the relevant region, followed by sequencing of the PCR product. From the sequence, the presence of the SNP and homo- or heterozygosity was determined. Primers are available upon request.

\section{Results}

\section{Detection of enterovirus RNA in PBMC of T1D patients}

Ten recently diagnosed T1D patients were tested by RTPCR (9), and compared with 20 healthy children of the same age without known risk factors for T1D. PBMC and plasma were tested separately. Four of the 10 T1D patients $(40 \%)$ were positive for enterovirus in their PBMC, and two of them were also positive in plasma (Table 1). The outcome was confirmed on separately isolated total NA by real-time PCR (19), and by NASBA, an RNA-based amplification technique (data not shown). Amplification of part of the 5'UTR yielded a fragment that showed strong similarities to viruses from the human enterovirus B group. Unfortunately, attempts to amplify the variable region (VP1-2A) using degenerate primers failed, making further typing of the enteroviruses impossible. None of the 20 children in the control group were positive for enteroviral RNA in either PBMC or plasma (Table 1). All controls were sampled in June 2004, whereas T1D patients were sampled throughout the year. Although this could have led to bias, there was no obvious 
TAble 1. BACKground Information and Enterovirus RNA Detection in T1D Patients and Controls

\begin{tabular}{|c|c|c|c|c|c|c|c|c|}
\hline Patient & Date of birth & Gender & $H L A-D R B 1$ & Plasma & $P B M C$ & Feces & Throat & $I F N-\alpha$ \\
\hline 1 & 04-05-1998 & M & DRB1*04/DRB1*13 & + & + & + & - & $<$ \\
\hline 2 & 11-05-1989 & M & DRB1*0301/DRB1*04 & - & - & - & - & $<$ \\
\hline 3 & 02-08-1992 & $\mathrm{M}$ & DRB1*01/DRB1*13 & - & - & - & - & 13 \\
\hline 4 & 30-05-1995 & M & DRB1*0301/DRB1*04 & - & + & - & - & $<$ \\
\hline 5 & 22-03-1996 & $\mathrm{F}$ & DRB1*04/DRB1*15 & - & - & - & - & $<$ \\
\hline 6 & 29-11-1995 & M & DRB1*01/DRB1*0301 & - & - & n.t. & - & $<$ \\
\hline 7 & 31-08-1993 & $\mathrm{F}$ & DRB1*04/DRB1*10 & - & + & n.t. & - & $<$ \\
\hline 8 & 03-03-1995 & $\mathrm{F}$ & DRB1*01/DRB1*04 & - & - & - & - & $<$ \\
\hline 9 & 11-07-1995 & $\mathrm{F}$ & DRB1*04/DRB1*08 & - & - & - & - & n.t. \\
\hline 10 & 04-02-1993 & $\mathrm{F}$ & DRB1*04/DRB1*04 & + & + & - & - & n.t. \\
\hline Control & Date of birth & Gender & $H L A-D R B 1$ & Plasma & PBMC & Feces & Throat & $I F N-\alpha$ \\
\hline 1 & 03-07-1998 & $\mathrm{F}$ & DRB1*14/DRB1*15 & - & - & n.t. & - & $<$ \\
\hline 2 & 06-03-1997 & M & DRB1*0301/DRB1*11 & - & - & n.t. & - & $>500$ \\
\hline 3 & 20-05-1989 & $\mathrm{F}$ & DRB1*04/DRB1*11 & - & - & n.t. & - & $<$ \\
\hline 4 & $15-08-1988$ & M & DRB1*13/DRB1*14 & - & - & n.t. & - & $<$ \\
\hline 5 & 25-05-1992 & $\mathrm{F}$ & DRB1*04/DRB1*13 & - & - & n.t. & - & $<$ \\
\hline 6 & 01-02-1989 & M & DRB1*07/DRB1*07 & - & - & n.t. & - & $<$ \\
\hline 7 & 23-05-1995 & M & DRB1*01/DRB1*04 & - & - & n.t. & - & $<$ \\
\hline 8 & 29-09-1989 & M & DRB1*14/DRB1*14 & - & - & n.t. & - & $<$ \\
\hline 9 & 07-02-1996 & $\mathrm{M}$ & DRB1*04/DRB1*15 & - & - & n.t. & - & $<$ \\
\hline 10 & 29-01-1993 & M & DRB1*01/DRB1*13 & - & - & n.t. & - & $<$ \\
\hline 11 & 17-02-1997 & $?$ & DRB1*01/DRB1*04 & - & - & n.t. & - & $<$ \\
\hline 12 & 17-06-1991 & $\mathrm{F}$ & DRB1*04/DRB1*14 & - & - & n.t. & - & $<$ \\
\hline 13 & 27-03-1993 & $\mathrm{F}$ & DRB1*07/DRB1*13 & - & - & n.t. & - & $<$ \\
\hline 14 & $10-09-1989$ & M & DRB1*04/DRB1*11 & - & - & n.t. & - & 75 \\
\hline 15 & $30-08-1993$ & $\mathrm{~F}$ & DRB1*01/DRB1*0301 & - & - & n.t. & - & $<$ \\
\hline 16 & 19-04-1989 & $\mathrm{F}$ & DRB1*01/DRB1*04 & - & - & n.t. & - & $<$ \\
\hline 17 & 08-01-1987 & $\mathrm{F}$ & DRB1*14/DRB1*15 & - & - & n.t. & - & $<$ \\
\hline 18 & 10-09-1988 & $\mathrm{F}$ & DRB1*0302/DRB1*07 & - & - & n.t. & - & $<$ \\
\hline 19 & 04-10-1988 & $\mathrm{M}$ & DRB1*04/DRB1*13 & - & - & n.t. & - & $<$ \\
\hline 20 & $15-03-1988$ & $\mathrm{~F}$ & DRB1*01/DRB1*0301 & - & - & n.t. & - & $<$ \\
\hline
\end{tabular}

Abbreviations: $\mathrm{M}$, male; $\mathrm{F}$, female; ?, gender unknown; +, positive reaction; -, negative reaction; n.t., not tested. Interferon- $\alpha($ IFN- $\alpha$ ) plasma levels given in $\mathrm{pg} / \mathrm{mL}$; detection threshold $10 \mathrm{pg} / \mathrm{mL} ;<$, less than the detection limit.

tendency of the four positive patients to test positive in any particular season, and only one of the four was sampled in the "enterovirus season," late summer and early autumn. The virus-positive T1D patients were sampled in August, November (2), and February.

\section{Prolonged enterovirus infection in T1D patients}

Little is known about the stage of enterovirus infection in T1D patients that are enterovirus-positive at the onset of disease. Therefore we investigated whether virus could be detected in throat or stool samples, which may have given some indication of the stage of infection. Throat and stool samples were collected at the same time as the blood samples were drawn. The samples were tested by RT-PCR (throat) or virus isolation and NASBA (fecal samples). The throat samples were invariably negative, as was virus isolation, whereas enterovirus was detected by NASBA in only 1 of the 4 virus-positive patients, suggesting that the infections were in a late stage or of prolonged duration (4) (Table 1).

\section{Detection of IFN- $\alpha$}

In a previous study researchers found that T1D patients have elevated levels of IFN- $\alpha$ in their blood, which correlated with the presence of enterovirus (3). Using ELISA, we in- vestigated whether the presence of viral RNA was linked to increased IFN- $\alpha$ levels in the blood, but no association was found (Table 1).

\section{Enterovirus and genetic risk factors}

The prolonged presence of viral RNA in PBMC of T1D patients suggests some intrinsic susceptibility to enterovirus infection or an inability to clear the infection. Some of the genetic risk factors that predispose to T1D also play a role in antiviral defense, and thus may be involved in aberrant clearance of enteroviruses. Therefore we tested whether the presence of viral RNA was linked with HLA-DR type and/or with SNPs in either MDA5 or OAS1.

HLA typing was performed on DNA isolated from PBMC. The T1D-predisposing HLA-DR4 and to a lesser extent DR3 were more abundant in the T1D population: 9 of the $10(90 \%)$ T1D patients had a DR3 and/or DR4 allele, versus 13 out of $20(65 \%)$ in the control group (Table 1). The high-risk DR3/DR4 or DR4/DR4 phenotype was found in 3 of 10 (30\%) T1D patients: 2 of $4(50 \%)$ virus-positive patients, versus 1 of $6(17 \%)$ virus-negative patients, but not in any of the 20 controls. All virus-positive patients had a DR4 allele.

The MDA5 and OAS1 polymorphisms were assessed by amplification of the region containing the SNPs, followed by 
sequencing of the PCR product. Smyth et al. found a higher prevalence of AA and GA in MDA5 in T1D patients (AA + GA $87.4 \%$ in T1D patients and $85.5 \%$ in controls) (26). We found that the T1D-associated AA and GA genotypes in MDA 5 were present in 3 and 6 of 10 patients $(90 \%)$ and in 5 and 12 of 20 controls (85\%), respectively. Heterozygosity was most prominent in virus-positive patients ( 3 of 4 or $75 \%$ ) (Table 2). The remaining virus-positive patient had an AA genotype. Three out of six virus-negative patients $(50 \%)$ had a GA genotype, $2(33 \%)$ had a GG genotype, and $1(17 \%)$ an AA genotype (Table 2). Thus no major differences were observed in the MDA5 polymorphism between virus-positive and virus-negative patients.

Field and colleagues reported an increased prevalence of GG and GA in OAS1 in the T1D group (GG + GA 58.7\% of T1D patients, compared to $50.3 \%$ of controls) (11). However, Smyth et al. did not find an increased prevalence of GG and GA genotypes in T1D patients (27). We found that T1Dassociated GG and GA genotypes of OAS1 were present in 2 and 3 of 10 T1D patients (50\%) and in 3 and 8 out of 20 controls $(55 \%)$, respectively (Table 2$)$. Thus no obvious differences were observed between T1D patients and the control group. Remarkably, only 1 of $4(25 \%)$ virus-positive patients had a GG or GA genotype, compared to 4 out of 6

TAble 2. SNP Genotypes of OAS1 AND MDA5 POLYMORPHISMS

\begin{tabular}{|c|c|c|c|}
\hline Patient & Virus & MDA5 & OAS1 \\
\hline 1 & + & GA & $\mathrm{AA}$ \\
\hline 2 & - & GA & GA \\
\hline 3 & - & GG & GA \\
\hline 4 & + & AA & AA \\
\hline 5 & - & AA & GG \\
\hline 6 & - & GA & $\mathrm{AA}$ \\
\hline 7 & + & GA & GG \\
\hline 8 & - & GA & GA \\
\hline 9 & - & AA & $\mathrm{AA}$ \\
\hline 10 & + & GA & $\mathrm{AA}$ \\
\hline Control & Virus & MDA5 & OAS1 \\
\hline 1 & - & GG & $\mathrm{AA}$ \\
\hline 2 & - & $\mathrm{AA}$ & GA \\
\hline 3 & - & GA & GA \\
\hline 4 & - & GA & GA \\
\hline 5 & - & $\mathrm{AA}$ & GG \\
\hline 6 & - & AA & GA \\
\hline 7 & - & GG & GA \\
\hline 8 & - & AA & $\mathrm{AA}$ \\
\hline 9 & - & GA & GA \\
\hline 10 & - & GA & $\mathrm{AA}$ \\
\hline 11 & - & GA & GA \\
\hline 12 & - & GA & $\mathrm{AA}$ \\
\hline 13 & - & GA & $\mathrm{AA}$ \\
\hline 14 & - & GA & GG \\
\hline 15 & - & GA & $\mathrm{AA}$ \\
\hline 16 & - & AA & GA \\
\hline 17 & - & GA & $\mathrm{AA}$ \\
\hline 18 & - & GA & $\mathrm{AA}$ \\
\hline 19 & - & GG & GG \\
\hline 20 & - & GA & $\mathrm{AA}$ \\
\hline
\end{tabular}

Abbreviations: + , enterovirus-positive; -, enterovirus-negative.
(67\%) virus-negative T1D patients (Table 2). Ours was a small sample, and future studies in which more T1D patients are included are required to shed more light on the association of MDA5 and OAS1 SNPs with T1D, and with the presence of viral RNA in PBMCs of T1D patients.

\section{Discussion}

In this study we demonstrated the presence of enterovirus RNA in the blood of 4 out of 10 T1D patients early after onset. Viral RNA was mainly detected in PBMC, the throat samples were negative, and only in 1 case was the stool also positive. An HLA-DR4 allele was present in all enteroviruspositive patients, yet no obvious link with polymorphisms in MDA5 or OAS1 was found.

Four out of $10 \mathrm{~T} 1 \mathrm{D}$ patients were found to be positive for enterovirus RNA in PBMCs. We used very sensitive methods for virus detection; levels as low as $0.002-0.01$ TCID $_{50}$ were detectable with the PCR and NASBA tests we used, depending on the enterovirus strain. All patient samples were tested with two different PCRs and NASBA to confirm the results. However, we cannot conclude that the 6 virusnegative T1D patients had no RNA in their PBMCs, as their levels may have been below the detection limit of the tests we used.

The finding of viral RNA in the blood of T1D patients is in accordance with results of previous studies $(1,3,5,6,17,18,32)$. In most studies, researchers tested for the presence of enteroviral RNA in whole blood, plasma, or serum samples. To date, in only one study was the presence of enteroviral RNA specifically sought in PBMCs of T1D patients (32). Our study shows that viral RNA is mainly present in the fraction of PBMCs, as only 2 out of 4 PBMC-positive patients were also positive in plasma. This indicates that the PBMC fraction may be the most important fraction to consider when screening for enterovirus positivity in human blood. That viral RNA was mainly detected in PBMCs suggests either infection of cells of the immune system, or uptake of virusinfected cells or cell-debris (e.g., by dendritic cells, as we demonstrated in vitro [14]). Infection of immune cells versus uptake of virus-containing material may have different consequences for immune function (impairment versus immune system activation). Whether and how altered immune homeostasis in the blood may affect immune homeostasis in the pancreas, thereby contributing to the development of T1D, requires further investigation.

During the early phase of infection, enterovirus is excreted in the throat and gastrointestinal tract. In this phase, the virus can sometimes also be detected in blood, but this is highly unusual, and occurs primarily during severe infections $(12,31)$. We found no enterovirus RNA in the blood of healthy controls, a finding that also corresponds with other reports, in which no or very few controls were found to be enterovirus RNA-positive in the blood $(1,3,5,17,18)$. During convalescence, virus is only detected in the stool, and fecal excretion can last for up to 3 mo (4). All throat and 3 of 4 stool samples were negative in the 4 T1D patients that were positive in PBMCs, arguing against acute infection. Whether this indicates a late stage of infection, or even a prolonged infection, remains to be determined.

The occurrence of prolonged enterovirus infection in T1D patients has also been suggested by Oikarinen and co- 
workers (21), who provided evidence for ongoing enteroviral infection in the gut mucosa of T1D patients. Detection of enterovirus antigens and RNA in pancreatic islets from T1D patients also suggests inadequate clearance $(10,23,33)$.

Assuming that T1D patients or individuals at risk for T1D have delayed clearance of enteroviruses, the question arises as to whether and which genetic factors predispose to it. We selected genetic risk factors for T1D that play a role in antiviral defense and investigated their linkage with the presence of virus. The most obvious risk factors are HLA-DR and molecules of the IFN pathway. It was to be expected that an association would be found with the well-known high-risk alleles DR3/DR4 and DR4/DR4, but no link with one of these phenotypes was found; DR4 was present in all 4 viruspositive patients. In a previous study, researchers reported impaired cellular immune reactivity against coxsackie B virus 4 in T1D patients, but this was not associated with either HLA-DR3 or HLA-DR4 (25). Published data are inconsistent with regard to HLA and virus positivity: Andreoletti et al. found DR3/DR4 in 2 out of 6 virus-positive T1D patients and 3 carrying a DR3 allele (1), whereas Craig et al. reported that DR3 was significantly less prevalent in viruspositive T1D patients (6). Clearly, further studies are required to address these associations, but it appears unlikely that a link with viral presence will be found.

Among the other genetic risk factors, MDA5 and OAS1 are of great interest, because they are involved in the innate response against RNA viruses, such as enteroviruses. No major differences were found between the T1D patients and controls, or between virus-positive and virus-negative T1D patients. Again, a link here seems unlikely, but our sample size was too small to say so definitively.

\section{Conclusion}

In conclusion, our data indicate that T1D patients may have delayed enterovirus clearance, with virus detected predominantly in PBMC. No obvious link was found with polymorphisms in MDA5 or OAS1, yet HLA-DR4 was present in all enterovirus-positive patients. Further studies are required to elucidate the intriguing relationship between enterovirus infection, genetic determinants of antiviral immunity, and the risk of acquiring T1D.

\section{Acknowledgments}

The authors thank Kjeld van Houwelingen (Department of Bloodtransfusion and Transplantation Immunology, Radboud University Nijmegen Medical Centre, Nijmegen) for help with HLA-DR typing, and Annemieke Boot (Sophia Children's Hospital, Erasmus Medical Centre, Rotterdam) for assistance with sample collection.

\section{Author Disclosure Statement}

No competing financial interests exist.

\section{Acknowledgments}

This study was supported by the Dutch Diabetes Research Foundation (pilot grant 2002.11.003), the Juvenile Diabetes Research Foundation (grant number 24-2008-949), and a grant from the Radboud University Nijmegen Medical Centre (grant number 2005-8).

\section{References}

1. Andreoletti L, Hober D, Hober-Vandenberghe C, Belaich S, Vantyghem MC, Lefebvre J, and Wattre P: Detection of coxsackie B virus RNA sequences in whole blood samples from adult patients at the onset of type I diabetes mellitus. J Med Virol 1997;52:121-127.

2. Atkinson MA, and Eisenbarth GS: Type 1 diabetes: new perspectives on disease pathogenesis and treatment. Lancet 2001;358:221-229.

3. Chehadeh W, Weill J, Vantyghem MC, Alm G, Lefebvre J, Wattre $\mathrm{P}$, and Hober D: Increased level of interferon-alpha in blood of patients with insulin-dependent diabetes mellitus: relationship with coxsackievirus B infection. J Infect Dis 2000;181:1929-1939.

4. Chung PW, Huang YC, Chang LY, Lin TY, and Ning HC: Duration of enterovirus shedding in stool. J Microbiol Immunol Infect 2001;34:167-170.

5. Clements GB, Galbraith DN, and Taylor KW: Coxsackie B virus infection and onset of childhood diabetes. Lancet 1995;346:221-223.

6. Craig ME, Howard NJ, Silink M, and Rawlinson WD: Reduced frequency of HLA DRB1*03-DQB1*02 in children with type 1 diabetes associated with enterovirus RNA. J Infect Dis 2003;187:1562-1570.

7. Cucca F, Lampis R, Congia M, et al.: A correlation between the relative predisposition of MHC class II alleles to type 1 diabetes and the structure of their proteins. Hum Mol Genet 2001;10:2025-2037.

8. Dahlquist GG, Forsberg J, Hagenfeldt L, Boman J, and Juto $\mathrm{P}$ : Increased prevalence of enteroviral RNA in blood spots from newborn children who later developed type 1 diabetes: a population-based case-control study. Diabetes Care 2004; 27:285-286.

9. de Leeuw N, Melchers WJ, Balk AH, de Jonge N, and Galama JM: No evidence for persistent enterovirus infection in patients with end-stage idiopathic dilated cardiomyopathy. J Infect Dis 1998;178:256-259.

10. Dotta F, Censini S, van Halteren AG, et al.: Coxsackie B4 virus infection of beta cells and natural killer cell insulitis in recent-onset type 1 diabetic patients. Proc Natl Acad Sci USA 2007;104:5115-5120.

11. Field LL, Bonnevie-Nielsen V, Pociot F, Lu S, Nielsen TB, and Beck-Nielsen H: OAS1 splice site polymorphism controlling antiviral enzyme activity influences susceptibility to type 1 diabetes. Diabetes 2005;54:1588-1591.

12. Galama JMD: Enteroviral infections in the immunocompromised host. Rev Med Microbiol 1997;8:33-40

13. Hyoty H, and Taylor KW: The role of viruses in human diabetes. Diabetologia 2002;45:1353-1361.

14. Kramer M, Schulte BM, Toonen LW, et al.: Phagocytosis of picornavirus-infected cells induces an RNA-dependent antiviral state in human dendritic cells. J Virol 2008;82:29302937.

15. Lonnrot M, Korpela K, Knip M, et al.: Enterovirus infection as a risk factor for beta-cell autoimmunity in a prospectively observed birth cohort: the Finnish Diabetes Prediction and Prevention Study. Diabetes 2000;49:1314-1318.

16. Lonnrot M, Salminen $K$, Knip M, et al.: Enterovirus RNA in serum is a risk factor for beta-cell autoimmunity and clinical type 1 diabetes: a prospective study. Childhood Diabetes in Finland (DiMe) Study Group. J Med Virol 2000;61:214-220.

17. Moya-Suri V, Schlosser M, Zimmermann K, Rjasanowski I, Gurtler L, and Mentel R: Enterovirus RNA sequences in sera of schoolchildren in the general population and their 
association with type 1-diabetes-associated autoantibodies. J Med Microbiol 2005;54:879-883.

18. Nairn C, Galbraith DN, Taylor KW, and Clements GB: Enterovirus variants in the serum of children at the onset of type 1 diabetes mellitus. Diabetes Med 1999;16:509-513.

19. Nijhuis M, van Maarseveen N, Schuurman R, Verkuijlen S, de Vos M, Hendriksen K, and van Loon AM: Rapid and sensitive routine detection of all members of the genus enterovirus in different clinical specimens by real-time PCR. J Clin Microbiol 2002;40:3666-3670.

20. Noble JA, Valdes AM, Cook M, Klitz W, Thomson G, and Erlich HA: The role of HLA class II genes in insulindependent diabetes mellitus: molecular analysis of 180 Caucasian, multiplex families. Am J Hum Genet 1996;59: 1134-1148.

21. Oikarinen M, Tauriainen S, Honkanen T, et al.: Detection of enteroviruses in the intestine of type 1 diabetic patients. Clin Exp Immunol 2008;151:71-75.

22. Ounissi-Benkalha $\mathrm{H}$, and Polychronakos $\mathrm{C}$ : The molecular genetics of type 1 diabetes: new genes and emerging mechanisms. Trends Mol Med 2008;14:268-275.

23. Richardson SJ, Willcox A, Bone AJ, Foulis AK, and Morgan NG: The prevalence of enteroviral capsid protein vp1 immunostaining in pancreatic islets in human type 1 diabetes. Diabetologia 2009;52:1143-1151.

24. Roivainen M: Enteroviruses: new findings on the role of enteroviruses in type 1 diabetes. Int J Biochem Cell Biol 2006;38:721-725.

25. Skarsvik S, Puranen J, Honkanen J, et al.: Decreased in vitro type 1 immune response against coxsackie virus B4 in children with type 1 diabetes. Diabetes 2006;55:996-1003.

26. Smyth DJ, Cooper JD, Bailey R, et al.: A genome-wide association study of nonsynonymous SNPs identifies a type 1 diabetes locus in the interferon-induced helicase (IFIH1) region. Nat Genet 2006;38:617-619.

27. Smyth DJ, Cooper JD, Lowe CE, Nutland S, Walker NM, Clayton DG, and Todd JA: No evidence for association of
OAS1 with type 1 diabetes in unaffected siblings or type 1 diabetic cases. Diabetes 2006;55:1525-1528.

28. Tessier MC, Qu HQ, Frechette R, et al.: Type 1 diabetes and the OAS gene cluster: association with splicing polymorphism or haplotype? J Med Genet 2006;43:129-132.

29. Thomson G, Robinson WP, Kuhner MK, et al.: Genetic heterogeneity, modes of inheritance, and risk estimates for a joint study of Caucasians with insulin-dependent diabetes mellitus. Am J Hum Genet 1988;43:799-816.

30. Todd JA, Walker NM, Cooper JD, et al.: Robust associations of four new chromosome regions from genome-wide analyses of type 1 diabetes. Nat Genet 2007;39:857-864.

31. Welch JB, McGowan K, Searle B, Gillon J, Jarvis LM, and Simmonds P: Detection of enterovirus viraemia in blood donors. Vox Sang 2001;80:211-215.

32. Yin H, Berg AK, Tuvemo T, and Frisk G: Enterovirus RNA is found in peripheral blood mononuclear cells in a majority of type 1 diabetic children at onset. Diabetes 2002;51:19641971.

33. Ylipaasto $\mathrm{P}$, Klingel $\mathrm{K}$, Lindberg AM, Otonkoski T, Kandolf $\mathrm{R}$, Hovi T, and Roivainen M: Enterovirus infection in human pancreatic islet cells, islet tropism in vivo and receptor involvement in cultured islet beta cells. Diabetologia 2004; 47:225-239.

Address correspondence to: Dr. Barbara M. Schulte Department of Medical Microbiology Radboud University Nijmegen Medical Centre P.O. Box 9101, $6500 \mathrm{HB}$ Nijmegen, the Netherlands

E-mail: b.schulte@ncmls.ru.nl

Received August 7, 2009; accepted October 10, 2009. 